\title{
Identifying Dynamic Membrane Structures with Atomic-Force Microscopy and Confocal Imaging
}

\author{
Tobias Timmel, ${ }^{1, *}$ Markus Schuelke, ${ }^{2}$ and Simone Spuler ${ }^{1}$ \\ ${ }^{1}$ Muscle Research Unit, Experimental and Clinical Research Center, a joint cooperation between the Charité Medical Faculty \\ and the Max Delbrück Center for Molecular Medicine Berlin, Lindenberger Weg 80, D-13125 Berlin, Germany \\ ${ }^{2}$ Department of Neuropediatrics and NeuroCure Clinical Research Center, Charité Universitätsmedizin, Augustenburger Platz 1, \\ D-13353Berlin, Germany
}

\begin{abstract}
Combining the biological specificity of fluorescence microscopy with topographical features revealed by atomic force microscopy (AFM) provides new insights into cell biology. However, the lack of systematic alignment capabilities especially in scanning-tip AFM has limited the combined application approach as AFM drift leads to increasing image mismatch over time. We present an alignment correction method using the cantilever tip as a reference landmark. Since the precise tip position is known in both the fluorescence and AFM images, exact re-alignment becomes possible. We used beads to demonstrate the validity of the method in a complex artificial sample. We then extended this method to biological samples to depict membrane structures in fixed and living human fibroblasts. We were able to map nanoscale membrane structures, such as clathrin-coated pits, to their respective fluorescent spots. Reliable alignment between fluorescence signals and topographic structures opens possibilities to assess key biological processes at the cell surface such as endocytosis and exocytosis.
\end{abstract}

Key words: atomic force microscopy, confocal microscopy, fluorescence imaging, image alignment, caveolae, clathrin-coated pits

\section{INTRODUCTION}

The atomic force microscope (AFM) has emerged as a powerful tool in nanotechnology. Using a very sharp tip, it scans across the sample and provides topographical information with a very high precision at nanoscale resolution. AFM exploits the broad spectrum of tip-sample interaction schemes to measure binding forces, to assess sample elasticity, or to separate chemically distinct phases. The ease of sample preparation and the ability to image in liquid has made AFM a high-resolution imaging technique that competes with electron microscopy. However, a major drawback is the lack of specificity in determining the identity of surface structures and molecules. Optical microscopy, such as confocal laser scanning microscopy, provides specific chemical or biological information but is limited in resolution because of the diffraction barrier. Hence, AFM and optical microscopy are complementary techniques. Combining the strengths of both systems allows linking specific chemical or biological information to corresponding topographical features (Flores \& Toca-Herrera, 2009). Thus, it was possible to identify caveolae, small invaginations of the plasma membrane, by mapping fluorescent caveolin-1 spots to membrane pits in $\mathrm{CHO}$ cells (Lucius et al., 2003). On the cytoplasmic aspect of membranes, irregularly shaped domains corresponded to distinct fluorescent membrane receptors like immunoglobulin-E and GM1ganglioside (Frankel et al., 2006).

Received August 12, 2013; accepted December 16, 2013

${ }^{*}$ Corresponding author. tobias.timmel@charite.de
A major challenge in combined optical and AFM is the exact image alignment (Defranchi et al., 2005; Flores \& TocaHerrera, 2009). The challenges of proper alignment vary and depend on several factors such as scanner setup, software, and thermal drift. In scanning stage setups, the tip is aligned to the optical axis of the confocal microscope ensuring an inherent spatial correlation between topographical and optical information (Kassies et al., 2005). In contrast, tip scanners obtain topographical and optical information separately, as the tip moves across the spatially fixed sample. Thus, a proper image transformation considering nonlinear stretching, rotation, and offsetting of the images is necessary. Although AFM manufactures provide very reliable software suitable to overlay scanning and optical data, thermal drift remains a major problem. Thermal drift causes unpredictable bending of the cantilever (Müller et al., 1995) and a lateral drift of the AFM head, the sample, and the optical axis of the optical microscope. Despite initial image correlation and alignment, the lateral drift induces an increasing correlation mismatch over time reflected by an image displacement. While stage scanners may overcome this mismatch by repeated readjustment of the tip relative to the objective's optical axis (Kellermayer, 2011), raw data from tip scanners require adequate postprocessing to correlate the images. So far, image correlation has only been successfully applied to biological samples with coarse and repeated patterns that are easily recognizable by both imaging techniques (Frankel et al., 2006). Fluorescent beads are useful as points of reference in image correlation (Defranchi et al., 2005; Kellermayer, 2011) but they may detach, disappear, move on 
the biological surface, or impede the scanning process. We demonstrate a robust and easy method to correct the drift induced misalignment of previously correlated confocal fluorescent and AFM topographical images in scanning tip AFM setups.

\section{Materials and Methods}

\section{Cells, Cell Culture, and Transfection}

We cultured primary human fibroblasts lacking polymerase I and transcript release factor (PTRF) (Rajab et al., 2010) in DMEM (Gibco/Life Technologies Corp., Darmstadt, Germany). The medium contained $10 \%$ fetal calf serum (Gibco) and gentamycin $(40 \mu \mathrm{g} / \mathrm{mL}$, Gibco). On the day before transfection the cells were seeded onto culture dishes with a cover glass bottom (FD35; World Precision Instruments Inc., Berlin, Germany). We transfected the cells by microinjection using a FemtoJet system (Eppendorf, Hamburg, Germany) combined with the InjectMan NI2 (Eppendorf). The plasmid DNA used for the experiments was human clathrin light chain $\mathrm{A}(\mathrm{Clc})$ cloned into the pEGFP-C3 vector (Clontech Laboratories Inc., Mountain View, CA, USA), human caveolin-1 cloned into pTagRFP-C vector (Evrogen, Moscow, Russia), and human PTRF cloned into pEGFP-N3 vector (Clontech Laboratories Inc.). We diluted the plasmid DNA in ultrapure water to a final concentration of 50,150 , and $50 \mu \mathrm{g} / \mathrm{mL}$, respectively. After centrifugation at $4^{\circ} \mathrm{C}$ for 30 min we loaded the supernatant immediately onto the capillaries (Femtotip II; Eppendorf). Microinjections into the cytoplasm of the fibroblasts were done with an injection pressure of 150 to $170 \mathrm{hPa}$. One day after microinjection we controlled protein expression by EGFP and RFP epifluorescence, respectively.

\section{Microscopy and Image Processing}

We used a Nanowizard II AFM (JPK Instruments, Berlin, Germany) mounted to a confocal laser-scanning microscope (LSM 700; Zeiss, Göttingen, Germany). Before each experiment we applied the thermal noise method to calibrate the cantilever (Hutter \& Bechhoefer, 1993). The AFM data were processed with JPK Data Processing 4.2 software (JPK Instruments) and Gwyddion software (Necas \& Klapetek, 2012). Very few isolated streaks were removed by line interpolation. We enhanced the image contrast by slope correction resulting in image flattening. A polynomial was fitted and subtracted from each scan line independently or from the entire height image where appropriate. We additionally applied high-pass filtering to the height data of cells to further enhance structures having a higher spatial frequency (Kienberger et al., 2006).

For fluorescence imaging, we used the Zeiss LSM 700 confocal microscope equipped with a LCI Plan-Neofluar $63 \times / 1.3$ glycerol immersion lens. The fluorescence images were processed with the ImageJ Fiji distribution (Schindelin et al., 2012) adjusting brightness and contrast, removing background noise, and applying Gaussian smoothing.
We determined the particle sizes in the raw images measuring the full width at half maximum. Background transparency was set using Gimp software (www.gimp.org). Final assembly of the aligned images was done using Adobe Illustrator software (Adobe Systems Inc., San Jose, CA, USA).

We quantified the drift using a single particle tracking procedure in a time series of beads. We chose one bead for tracking in both the fluorescence and height images. We also tracked the cantilever tip in the fluorescence images. Tracking was performed using the MTrackJ plugin of the Fiji software package (Meijering et al., 2012). The tracked objects were localized with subpixel precision by centroid determination. The transform function to correlate optical and AFM images was calculated using the DirectOverlay module (JPK Instruments). From an image series of known tip positions the optical image was calibrated into the AFM coordinates accounting for nonlinear stretching, rotation, and offsetting. Once the system was optically calibrated, thermal drift caused an increasing lateral image shift over time. Readjustment of the image alignment was done by hand using the cantilever tip as landmark.

When translucent cantilevers without coating were used, the tip could clearly be localized in the bright-field image. Alternatively, we functionalized the cantilever tips using a biotin-streptavidin binding assay (Barattin \& Voyer, 2011). Cantilevers were cleaned in Piranha solution (3:1 mixture of sulfuric acid and 30\% hydrogen peroxide) and thoroughly rinsed in ultrapure water. Subsequently, they were incubated for $90 \mathrm{~min}$ at $37^{\circ} \mathrm{C}$ in biotin-BSA (SigmaAldrich $\mathrm{GmbH}$, Munich, Germany), diluted at $1 \mathrm{mg} / \mathrm{mL}$ in phosphate buffered saline (PBS, Gibco/Life Technologies Corp.). After washing the cantilevers in PBS, we incubated them for $30 \mathrm{~min}$ at room temperature in an Alexa Fluor 488 streptavidin conjugate (1 mg/mL in PBS; Molecular Probes/ Life Technologies Corp.). Next, we washed the cantilevers in PBS and stored them at $4^{\circ} \mathrm{C}$ until use.

\section{Imaging Beads}

Single fluorescent polystyrene microspheres (Bangs Laboratories Inc., Fishers, IN, USA) dyed with Flash Red served as test samples. The beads, having a diameter of $0.19 \mu \mathrm{m}$, were diluted in PBS containing 5\% bovine serum albumin (BSA; Carl Roth GmbH, Karlsruhe, Germany) to a final concentration of 1:10,000. A $100 \mu \mathrm{L}$ droplet of this suspension was dispersed on the glass bottom of a culture dish (FD35; World Precision Instruments Inc.) and dried at room temperature. AFM imaging was performed in contact mode under air. We used a functionalized silicon nitride cantilever with a silicon tip (Hydra 6V-200NG; Applied NanoStructures Inc., Mountain View, CA, USA). Its nominal spring constant was $0.045 \mathrm{~N} / \mathrm{m}$ and the resonant frequency $17 \mathrm{kHz}$. After determining the AFM scan region, the cantilever tip was lowered to the sample. Then the confocal microscope acquired a $z$-stack covering the very end of the fluorescent tip and the object of interest, the fluorescent beads. Subsequently, we started the AFM scan. The tip 
a
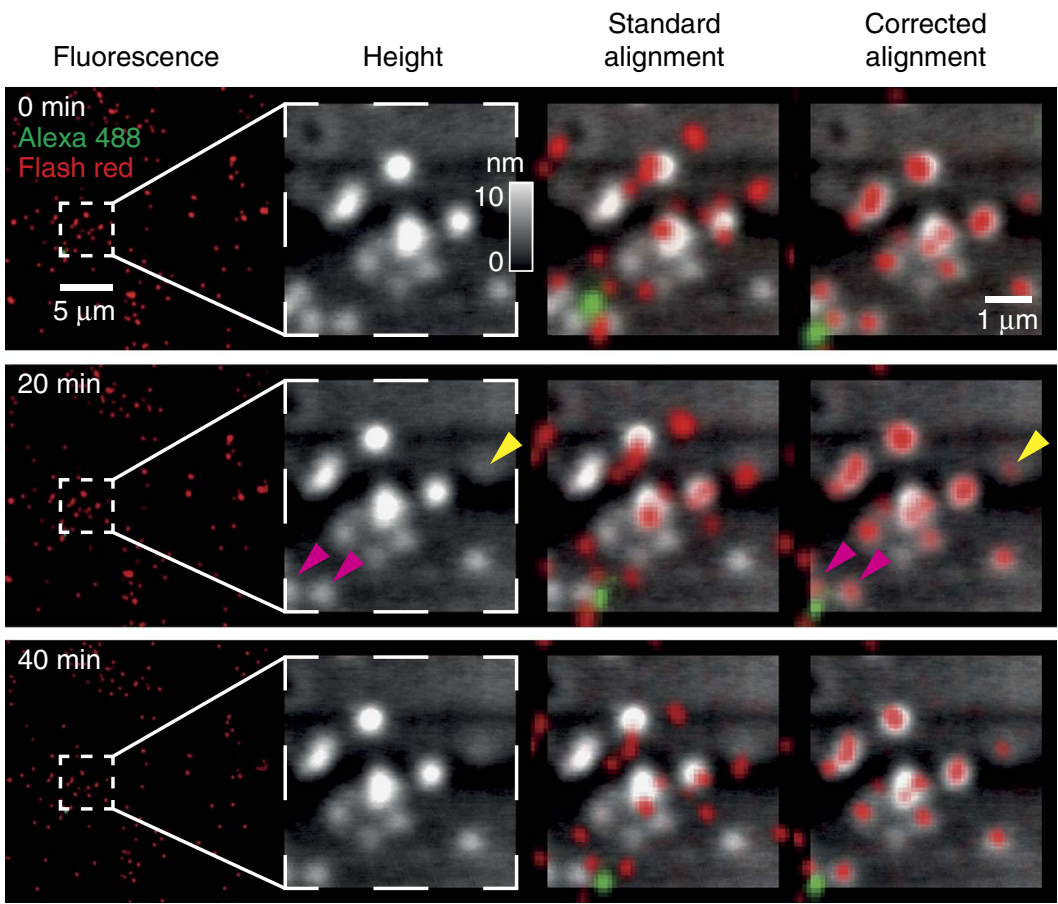

b
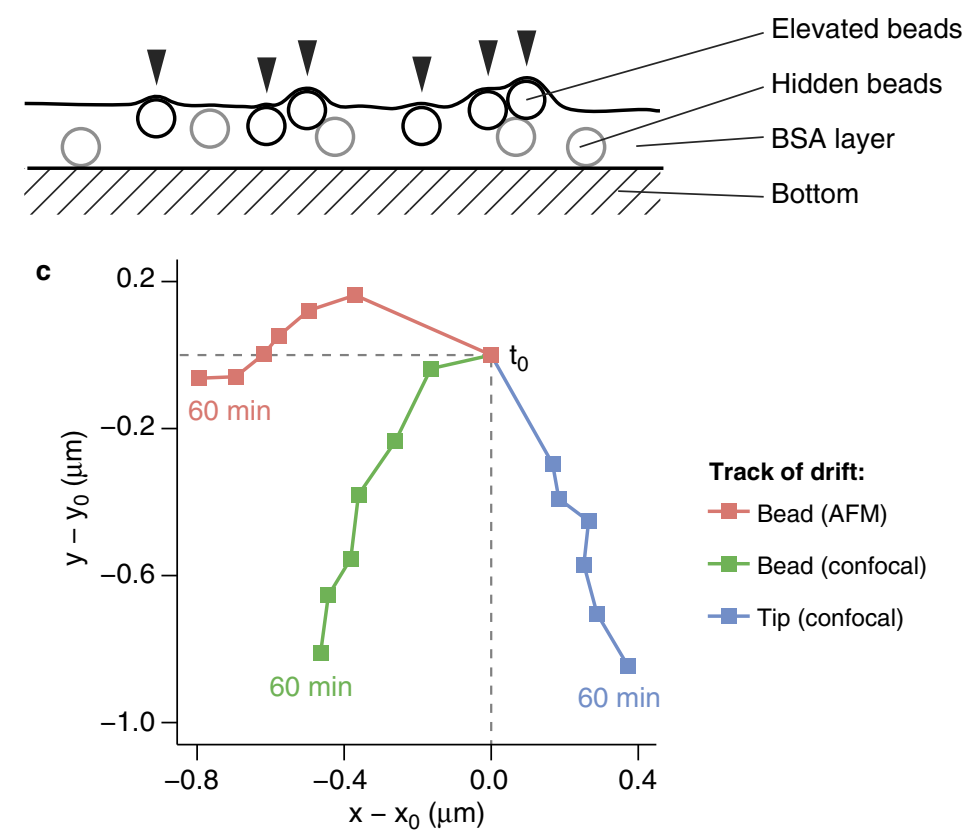

Figure 1. Complex artificial sample of fluorescent beads in a BSA layer imaged with combined confocal and atomic force microscopy (AFM). a: Time series of fluorescence (cantilever tip: green, beads: red), height, and overlay using the standard and the corrected alignment. The AFM scan region is depicted by a white rectangle. Several fluorescence spots correspond to elevations (magenta arrows) whereas others do not (yellow arrows). b: Schematic depiction of beads embedded in a BSA layer. Beads protruding from the BSA layer are detectable by AFM (arrows). c: $x-y$ plot of amount and direction of drift quantified in the time series of beads. Tracking paths are normalized such that the coordinates of the first frame $\left(t_{0}\right)$ were subtracted from each data point. Therefore, all tracks start at position $(0,0)$.

moved across the sample with a line rate of $2 \mathrm{~Hz}$ and a force setpoint of $4 \mathrm{nN}$. We acquired a time series of fluorescence and AFM images taken every $10 \mathrm{~min}$ over a period of $1 \mathrm{~h}$. Before each scan of the series we took a reference image of the cantilever tip.

\section{Imaging Cells}

For imaging fixed cells, fibroblasts were subjected to $3.7 \%$ formaldehyde-PBS solution for 12 min 1 day after microinjection. The AFM was operated in contact mode under liquid (PBS). Using a petri dish heater (JPK Instruments), 


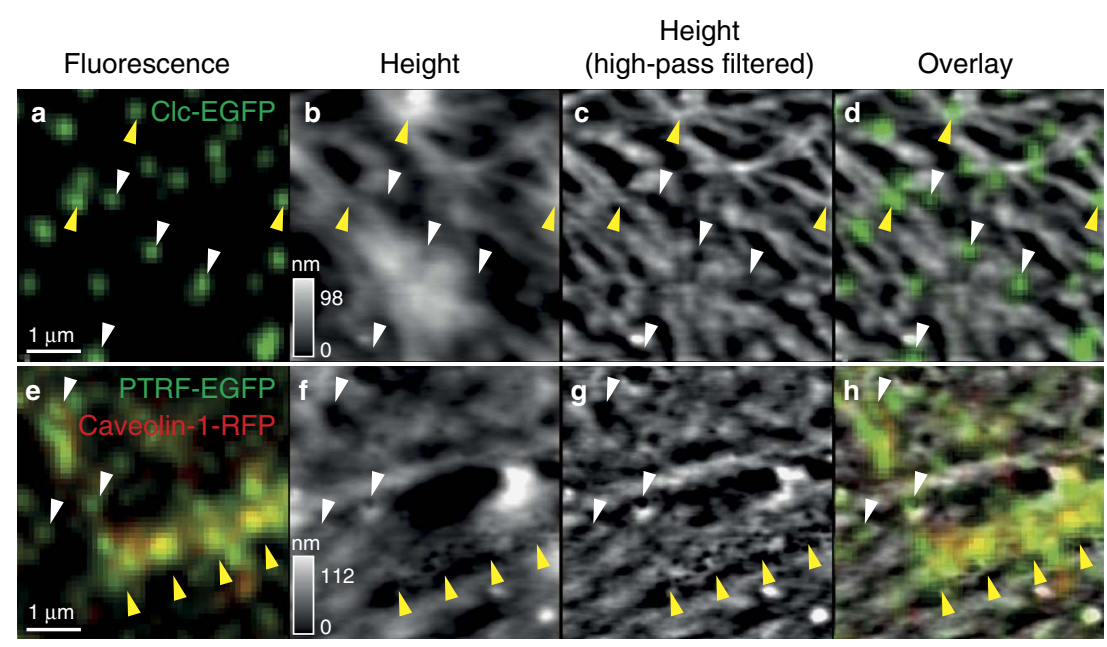

Figure 2. Fixed biological samples of primary human fibroblasts imaged with combined confocal and atomic force microscopy (AFM). Polymerase I and transcript release factor (PTRF)-deficient cells were transfected with Clc-EGFP (a-d) and with PTRF-EGFP and Caveolin-1-RFP (e-h). a, e: Fluorescence of Clc-EGFP (green) and of PTRF-EGFP (green) and caveolin-1-RFP (red), respectively. b, f: Flattened height images. c, g: High-pass filtered height images. d, e: Overlay of re-aligned high-pass filtered height and fluorescence. a-d: Many fluorescence spots of Clc-EGFP match depressions of the plasma membrane (white arrows). In contrast, some Clc-EGFP spots correspond to membrane protrusions (yellow arrows). e-h: Co-localizing PTRF-EGFP and caveolin-1-RFP that match depressions of the membrane are depicted by white arrows. The prominent dense fluorescence streak corresponding to a geometrically similar elongated and dense structure of membrane invaginations is depicted by yellow arrows.

we kept the temperature constant at $25^{\circ} \mathrm{C}$. A translucent soft silicon nitride cantilever with a silicon tip (Hydra 2R-100N, Applied NanoStructures Inc.) was chosen. This uncoated cantilever had a nominal spring constant of $0.011 \mathrm{~N} / \mathrm{m}$ and a resonant frequency of $21 \mathrm{kHz}$. Immediately after taking bright-field images of the cantilever tip and fluorescent images of the sample, we started the AFM scans. Several scans applying a force setpoint of $0.3 \mathrm{nN}$ at a line rate of $1 \mathrm{~Hz}$ were obtained. For live cell imaging, we set the temperature to $37^{\circ} \mathrm{C}$ and allowed the setup to thermally equilibrate for $\sim 2 \mathrm{~h}$. Before imaging the entire sequence, we obtained a bright-field image of the tip. We imaged the cells in Tyrode solution applying a force setpoint of $0.15 \mathrm{nN}$ at a line rate of $4-8 \mathrm{~Hz}$.

\section{Results And Discussion}

Our aim was to exactly align images obtained from unfixed biological samples by confocal laser scanning as well as by AFM. We describe a three-step approach. We first prepared a complex artificial sample that would not allow simple alignment through pattern recognition by eye. We embedded single fluorescent beads with a diameter of $200 \mathrm{~nm}$ in a layer of BSA with only a subset of the beads protruding from the layer that would become detectable for the AFM. Red fluorescent microspheres were dispersed in the culture dish in $5 \%$ BSA and dried. Drift induced image mismatch is demonstrated as a significant problem even after thermal equilibration and the DirectOverlay software tool was applied (Fig. 1a). We further analyzed the time series of beads using a particle tracking procedure. The tracking paths revealed different amounts and directions of drift of the sample, the scanner, and the optics being in the range of $1 \mu \mathrm{m} / \mathrm{h}$ each (Fig. 1c). We corrected the previously calculated transform function given by the DirectOverlay software by using the cantilever tip at the starting position of the AFM scan as a landmark. Since we could not localize the cantilever tip precisely in the bright-field image, we labeled it with fluorescent Alexa Fluor 488.

We were able to identify identical positions of fluorescent spots and height alterations in this model when combining confocal imaging and AFM. We also detected fluorescent spots that were undetectable by AFM because those beads were entirely embedded in the protein layer (Fig. 1a and 1b). One protrusion remained unstained. Despite the deviating punctate patterns between fluorescence and topography, the concentric position of associated fluorescence and height spots provides evidence for proper image alignment in the corrected overlay. The apparent size of the red beads in the fluorescence images was $\approx 300 \mathrm{~nm}$ reflecting the diffraction limited resolution of the confocal microscope. In the AFM, the corresponding protrusions had a height of $20 \mathrm{~nm}$ at the most and measured $300-400 \mathrm{~nm}$ in diameter indicating that the AFM overestimated the width of the beads. This state-of-affairs could be because of the BSA layer covering the beads. Moreover, the cantilever tip blurs the sample, and finally the tip functionalization using the biotin-streptavidin binding assay slightly increases the tip radius.

In our second setup we used human primary fibroblasts that were fixed with formaldehyde. We attempted to visualize clathrin-coated pits in fibroblasts deficient of PTRF (Rajab et al., 2010). PTRF-deficient fibroblasts cannot form 


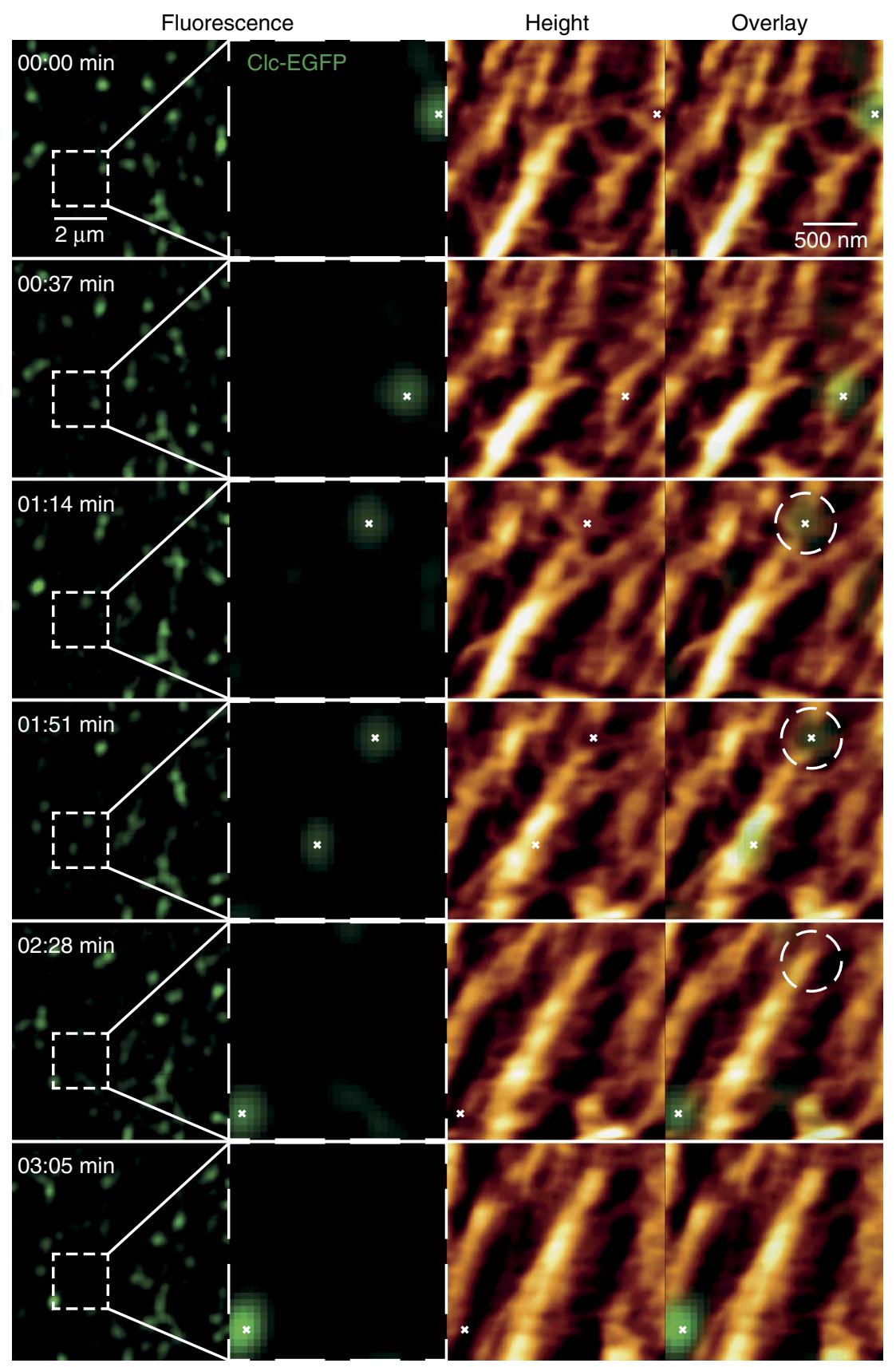

Figure 3. Time series of Clc-EGFP transfected living primary human fibroblasts showing EGFP fluorescence (green), height information, and the re-aligned overlay. The location of the AFM scan region is shown on the left (white rectangle). Spots of EGFP fluorescence are depicted by a white cross and drawn at identical image locations in the height and the overlay images. A vanishing Clc-EGFP spot that corresponds to a flattened pit is depicted by a white circle. The complete image sequence is shown in Supplementary Video S1. In Supplementary Video S2 we increased the frame rate to 20 s/image.

caveolae that are flask-shaped invaginations of the cell membrane about $50-100 \mathrm{~nm}$ in size. Thus, clathrin-coated pits, cell-membrane invaginations of similar size, were identified with confidence. Fibroblasts were transfected with EGFP tagged Clc. The fluorescence image of Clc-EGFP shows a punctate pattern of spots about $300 \mathrm{~nm}$ in size (Fig. 2a). The corresponding flattened (Fig. 2b) and additionally high-pass filtered AFM height image (Fig. 2c) revealed a highly corrugated surface. The high number of depressions makes a manual alignment by pattern comparison impossible. When we aligned fluorescence and topography using the tip of a nonfunctionalized cantilever as a landmark, many depressions co-localized with the Clc-EGFP signal and were interpreted as being clathrin-coated pits. However, we also detected protrusions that were superimposed Clc-EGFP signals. The association of membrane protrusions with clathrin-coated 
pits was previously shown by others in electron micrographs (Bretscher et al., 1980). Using scanning ion conductance microscopy in living COS-7 cells, other investigators recently observed that in $\approx 70 \%$ of clathrin-coated pits a protrusion formed shortly before pit closure (Shevchuk et al., 2012).

We also transfected PTRF-deficient fibroblasts with EGFP tagged PTRF and RFP tagged caveolin-1, which might serve as a sufficient signal to induce the formation of caveolae at the plasma membrane. Co-localization of PTRF and caveolin-1 therefore indicate the presence of caveolae (Fig. 2e) (Hill et al., 2008). Many depressions match the joint fluorescence of PTRF and caveolin-1 (Fig. 2f-2h). A prominent dense fluorescence streak can be mapped to a band of circular membrane pits having a diameter of $99 \pm 32 \mathrm{~nm}$. Caveolae may appear as arranged on a cord consistent with close association with microtubules and actin filaments (Bastiani \& Parton, 2010).

In the third setup, we attempted to track clathrin-coated pits in living human primary fibroblasts. We transfected PTRF-deficient fibroblasts with Clc-EGFP and obtained both fluorescence and AFM image series at an identical image acquisition rate. Before each image series we once localized the cantilever tip in the optical microscope and thus could reliably align fluorescence and AFM images. An image series with a scan rate of $37 \mathrm{~s}$ per image is shown in Figure 3 (Supplementary Video 1). Clc-EGFP fluorescence reveals a highly dynamic scenario with spots becoming visible, moving around, and disappearing. In agreement with the fixed samples some spots perfectly matched membrane depressions, whereas others mapped to protrusions. Moreover, when a Clc-EGFP spot vanished, in the next image the corresponding pit had also disappeared and flattened out suggesting that a clathrin-coated pit had pinched off the membrane (circles in Fig. 3). These kinetics lie within the time frame of pit lifetime as described by others (Loerke et al., 2009).

\section{Supplementary Video 1}

Supplementary Video 1 can be found online. Please visit journals.cambridge.org/jid_MAM.

We estimate the precision of tip localization around $50 \mathrm{~nm}$. This estimate is deduced from the diffraction-limited resolution of our microscope (Zeiss LSM 700), because the error mainly depends on the localization precision of the cantilever tip in the bright-field or fluorescence image. In the optical images the true tip coordinates are not known.

In our time series lasting $10-15 \mathrm{~min}$ alignment of the cantilever tip was necessary only once. We recommend repeated acquisition of reference frames for longer series. The required frequency of the alignment procedure depends on the experimental goals and conditions such as the ambient temperature, the available time to thermally equilibrate the setup, the targeted frame rate, and the total image acquisition period. Moreover, a completely automated procedure would be favorable in terms of imaging speed and temporal synchronization. Such an approach would require true integration of confocal and AFM setup controlled by a single software module. Although not synchronized, currently available AFM and confocal microscopes are capable of scripting routines allowing at least semi-automated image acquisition to further speed up imaging.

In the fixed samples, we acquired fluorescence and height images sequentially. In contrast, live cell images from both the confocal microscope and AFM were obtained simultaneously to increase the frame rate. However, potential scattering and luminescence from the cantilever tip might overwhelm the signal from single fluorophores (Gaiduk et al., 2005). Therefore, in weakly fluorescent samples, temporarily moving the cantilever tip out of the region of interest while obtaining the fluorophore signal of the sample might be preferable. Of course, this procedure requires sequential image acquisition of the tip location, the fluorescence of the sample, and the topography, and thus slightly decreases the frame rate.

\section{CONCLUSIONS}

We introduce an easy, reliable, and fast procedure to correct drift induced misalignment for combining confocal and scanning tip AFM imaging. Our method is suitable to monitor membrane dynamics of living cells at nanoscale dimensions. It opens up possibilities to assess biological key processes related to the cell's plasma membrane such as endocytosis and exocytosis.

\section{ACKNOWLEDGMENTS}

The German Research Foundation (DFG, clinical research group KFO-192, TP 7) supported the project. We thank Stefanie Meyer-Liesener and Stephanie Belz for excellent technical assistance. The Clc plasmid DNA was a gift from Lois E. Greene (NIH, USA) (Wu et al., 2001).

\section{ReFERENCES}

Barattin, R. \& Voyer, N. (2011). Chemical modifications of atomic force microscopy tips. Methods Mol Biol 736, 457-483.

Bastiani, M. \& Parton, R.G. (2010). Caveolae at a glance. J Cell Sci 123(Pt 22), 3831-3836.

Bretscher, M.S., Thomson, J.N. \& Pearse, B.M. (1980). Coated pits act as molecular filters. Proc Natl Acad Sci USA 77(7), 4156-4159.

Defranchi, E., Bonaccurso, E., Tedesco, M., Canato, M., Pavan, E., Ratteri, R. \& RegGiani, C. (2005). Imaging and elasticity measurements of the sarcolemma of fully differentiated skeletal muscle fibres. Microsc Res Tech 67(1), 27-35.

Flores, S.M. \& Toca-HerRera, J.L. (2009). The new future of scanning probe microscopy: Combining atomic force microscopy with other surface-sensitive techniques, optical microscopy and fluorescence techniques. Nanoscale 1(1), 40-49.

Frankel, D.J., Pfiiffer, J.R., Surviladze, Z., Johnson, A.E., Oliver, J.M., WiLSON, B.S. \& BuRNs, A.R. (2006). Revealing the topography of cellular membrane domains by combined atomic force microscopy/fluorescence imaging. Biophys J 90(7), 2404-2413. 
Gaiduk, A., Kühnemuth, R., Antonik, M. \& Seidel, C.A.M. (2005). Optical characteristics of atomic force microscopy tips for single-molecule fluorescence applications. Chemphyschem 6(5), 976-983.

Hill, M.M., Bastiani, M., Luetterforst, R., Kirkham, M., Kirkham, A., Nixon, S.J., Walser, P., Abankwa, D., Oorschot, V.M.J., Martin, S., Hancock, J.F. \& Parton, R.G. (2008). PTRF-Cavin, a conserved cytoplasmic protein required for caveola formation and function. Cell 132(1), 113-124.

Hutter, J.L. \& BechHoefer, J. (1993). Calibration of atomic-force microscope tips. Rev Sci Instrum 64(7), 1868-1873.

Kassies, R., van der Werf, K.O., Lenferink, A., Hunter, C.N., Olsen, J.D., Subramaniam, V. \& Otto, C. (2005). Combined AFM and confocal fluorescence microscope for applications in bio-nanotechnology. J Microsc 217(Pt 1), 109-116.

Kellermayer, M.S.Z. (2011). Combined atomic force microscopy and fluorescence microscopy. Methods Mol Biol 736, 439-456.

Kienberger, F., Pastushenko, V.P., Kada, G., Puntheeranurak, T., Chtcheglova, L., Riethmueller, C., Rankl, C., Ebner, A. \& Hinterdorfer, P. (2006). Improving the contrast of topographical AFM images by a simple averaging filter. Ultramicroscopy 106(8-9), 822-828.

Loerke, D., Mettlen, M., Yarar, D., Jaqaman, K., Jaqaman, H., Danuser, G. \& Schmid, S.L. (2009). Cargo and dynamin regulate clathrin-coated pit maturation. PLoS Biol 7(3), e57.

Lucius, H., Friedrichson, T., Kurzchalia, T.V. \& Lewin, G.R. (2003). Identification of caveolae-like structures on the surface of intact cells using scanning force microscopy. J Membr Biol 194(2), 97-108.
Meijering, E., Dzyubachyк, O. \& Smal, I. (2012). Methods for cell and particle tracking. Methods Enzymol 504, 183-200.

Müller, D.J., Schabert, F.A., Büldt, G. \& Engel, A. (1995). Imaging purple membranes in aqueous solutions at sub-nanometer resolution by atomic force microscopy. Biophys $J$ 68(5), 1681-1686.

Necas, D. \& Klapetek, P. (2012). Gwyddion: An open-source software for SPM data analysis. Cent Eu J Phys 10, 181-188.

Rajab, A., Straub, V., McCann, L.J., Sellow, D., Varon, R., Barresi, R., Schulze, A., Lucke, B., LÜtZKendorf, S., Karbasiyan, M., Bachmann, S., Spuler, S. \& Schuelke, M. (2010). Fatal cardiac arrhythmia and long-QT syndrome in a new form of congenital generalized lipodystrophy with muscle rippling (CGL4) due to PTRF-CAVIN mutations. PLoS Genet 6(3), e1000874.

Schindelin, J., Arganda-Carreras, I., Frise, E., Kaynig, V., Longair, M., Pietzsch, T., Preibisch, S., Rueden, C., SaAlfeld, S., Schmid, B., Tinevez, J.-Y., White, D.J., Hartenstein, V., Eliceiri, K., Tomancak, P. \& CARdona, A. (2012). Fiji: An open-source platform for biological-image analysis. Nat Methods 9(7), 676-682.

Shevchuk, A.I., Novak, P., Taylor, M., Diakonov, I.A., ZiyadehIsleem, A., Bitoun, M., Guicheney, P., Lab, M.J., GoreliK, J., Merrifield, C.J., Klenerman, D. \& Korchev, Y.E. (2012). An alternative mechanism of clathrin-coated pit closure revealed by ion conductance microscopy. J Cell Biol 197(4), 499-508.

Wu, X., Zhao, X., Baylor, L., Kaushal, S., Eisenberg, E. \& Greene, L.E. (2001). Clathrin exchange during clathrin-mediated endocytosis. J Cell Biol 155(2), 291-300. 